Norbert Richard Wolf

\title{
UNTERFRÄNKISCHE WORTGEOGRAPHIE ALS SPIEGEL DER KULTURGESCHICHTE
}

Unterfranken ist einer der sieben Regierungsbezirke des Freistaats Bayern; Sitz der Bezirksregierung und des Bezirkstags ist Würzburg. Im Süden und Südosten grenzen die R egierungsbezirke Mittel- und Oberfranken, im Südwesten, Westen und Norden die Bundesländer Baden-Württemberg, Hessen und Thüringen an Unterfranken. Die Dialekte in diesem Regierungsbezirk beschreibt das W ürzburger Forschungsprojekt ,Sprachatlas von Unterfranken' (SUF), und die Beispiele, die ich im Folgenden vorstellen werde, sind fertigen, aber noch nicht gedruckten Bänden des SUF entnommen. Nicht nur für mich ist U nterfranken der dialektologisch interessanteste Regierungsbezirk Bayerns, weil durch inn eine der wichtigsten Mundartgrenzen des Deutschen geht, die Germersheimer Linie bzw. die appel-apfel-Linie, die eine martialisch gestimmte Dialektgeografie früherer Zeiten auch Spessartbarriere genannt hat (vgl. insgesamt Karte 1 im Anhang).

Diese Grenze stimmt weitestgehend mit einer alten Territorialgrenze überein: Das Gebiet östlich der Spessartbarriere war das Hochstift Würzburg; der Teil westlich davon gehörte zum Erzstift Mainz; der dortige $\mathrm{H}$ auptort im heutigen Unterfranken, Aschaffenburg, war die Sommerresidenz der Mainzer Erzbischöfe.

Wie die Bezeichnung appel-apfel-Linie schon sagt, hat man mit dieser Grenze vor allem Isoglossen der althochdeutschen Konsonantenverschiebung verbunden. Doch Unterschiede zwischen dem östlichen Unterostfränkisch und dem westlichen R heinfränkisch (bzw. Hessisch) finden sich nicht nur in der Phonologie und der Morphologie, sondern ganz besonders auch im W ortschatz.

Das Wort Most gibt es in beiden Dialekträumen, und es bedeutet ,gegorener Fruchtsaft'. Doch im jeweiligen Areal bezeichnet dieses Wort Unterschiedliches (vgl. K I eiber 1996, Karte 109; Karte 2 im Anhang: ,Der W ein neuer Ernte'). 
Im Unterostfränkischen nennt man den Wein neuer Ernte Most, und zwar so lange, bis es neuen Wein neuer Ernte gibt. Im Rheinfränkischen hingegen bezeichnet M ost entweder den ungegorenen Fruchtsaft oder den Apfelwein, wie wir inn ja, besonders aus Frankfurt und Umgebung, auch aus einschlägigen Fernsehsendungen kennen. In zwei einschlägigen Wörterbüchern, die den rheinischen Usus buchen, finden wir s. v. M ost folgende Einträge:

Bad. Wb. (1997, 672):

1) Getränk.

a),unvergorener, frisch gekelterter Traubensaft' hauptsächlich in Weinbauorten am Oberrhein

b), vergorener Obstsaft aus Äpfeln und/oder Birnen' in ganz Baden verbreitet c), unvergorener Obstsaft vor dem V ergären' mancherorts auch eher süeße M ost

2) Traubensorte

Frankf. Wb. $(1988,2069)$

,gekelterter Saft von Trauben oder Obst‘

In den wenigen Orten westlich der Spessartbarriere, in denen ebenfalls W einbau betrieben wird, heißt der junge W ein junger Wein und eben nicht Most. Damit hängen weitere lexikalische Unterschiede zusammen (nach K I eiber 1996, K arte 105; siehe K arte 3 im Anhang):

Der gärende Saft wird im R heinfränkischen Federweißer und im Unterostfränkischen Bremser genannt.

Den neuen Wein nennt man übrigens ganz selten, östlich vom Mainviereck, also nahe der Dialektgrenze, Heuriger (vgl. Karte 1). Im Rheinfränkischen wurde junger Wein (zweimal am westlichen Mainviereck bei Erlenbach) erhoben, und sonst wurde dort überall neuer Wein notiert. Für das gesamte Untersuchungegebiet gilt, dass das Traubengetränk, sobald neuer W ein vorhanden ist, Wein in verschiedenen lautlichen Realisationen genannt wird (vgl. zum Ganzen K I eiber 1996, Karte 109).

W ir haben es hier mit einem interessanten lexikologischen Sachverhalt zu tun. Ein Wort, in unserem Fall Most, wird in zwei unterschiedlichen, wenngleich benachbarten Dialektarealen in gleicher Bedeutung, aber mit unterschiedlicher R eferenz gebraucht. Ich weiß nicht, ob man in solch einem F alle von ,referenzieller Homonymie' sprechen könnte. Diese Art von Homonymie wirkt sich auf weitere Bezeichnungen des Sachfeldes ,W ein' aus. Im Unterostfränkischen wird für den neuen Wein das Lexem Wein gemieden, während es im R heinfränkischen für den Traubenwein verwendet wird. 
Das W ort Most ist bekanntlich "aus I. mustum (vanum) n. ,junger W ein" zu I. mustus ,jung' " (K I uge 2002, S. 633) entlehnt. Für die Bedürfnisse im rheinfränkischen Teil Unterfrankens wurde es umgedeutet. Die speziellen semantischen Funktionen des Wortes Most sowie die unterschiedlichen Bezeichnungen für weitere Elemente dieses Sachfeldes resultieren somit aus unterschiedlichen ,Leitfrüchten' in unterschiedlichen Trinkkulturen. Im Territorium Würzburg wurde der Traubenwein schon im frühen Mittelalter ein bedeutender wirtschaftlicher Faktor, während die Landwirtschaft westlich des Spessarts sich auf andere F rüchte spezialisieren musste. Für das 16. J ahrhundert hat man, besonders für das Gebiet des Hochstifts Würzburg errechnet, dass die R ebfläche „etwa dem Zehnfachen des Weinbauareals kurz nach dem 2. Weltkrieg entsprochen hatte" (Schenk 1995, S. 569); im J ahre 1839 wurden 26.613 ha Weinbaufläche für ganz Bayern festgestellt, während es im 16. J ahrhundert etwa 40.000 ha nur in F ranken, und davon überwiegend im Gebiet östlich des Spessarts gewesen sein dürften. Im westlichen Teil des heutigen U nterfranken hingegen war der W einbau bei Weitem nicht so verbreitet, wenngleich für einige Städte am südwestlichen Mainviereck wie Miltenberg "W einbau und W einhandel die Grundlagen des städtischen Wohlstands" (F ischer 1995, S. 441) waren. W elche Rolle der W einbau im Hochstift Würzburg gerade auch in der frühen Neuzeit spielte, zeigt auch eine Plastik am so genannten N eutor zur W ürzburger Festung, die den Reichtum an W ein personifiziert (vgl. $K$ ol b/ K r enig 1999, S. 419).

Wir wissen zudem heute, dass im späten Mittelalter Territorialgrenzen häufig auch Kommunikationsgrenzen waren. Der Landesherr verfügte über die M obilität seiner Untertanen, und es gab auch V orschrift darüber, welches Wissen Z. B. ins ,Ausland' weiter gegeben werden durfte. Einen eindrucksvollen Fall schildert der Historiker Rolf Spr andel (1978, S. 267): „In einigen Fällen werden auch die Kommunikationsbarrieren an Staatsgrenzen mit Sanktionen versehen. 1478 schließen die Grafen von Nassau und die Grafen Sayn-Wittgenstein einen Vertrag darüber ab, daß die in ihren Ländern geübte Eisentechnik nicht über die Grenzen hinausdringen sollte. J eder Meister solle sich verpflichten, seine Kunst nicht über die Grenzen beider Länder hinauszutragen."

Auch wenn nicht viele solcher Beispiele bekannt sind, ist doch zu beachten, dass zwischen den beiden Territorien ein großes zusammenhängendes W aldgebiet lag und liegt, der Spessart. Dieses Gebiet war im späten Mittelalter und in der frühen Neuzeit nicht besiedelt; die Landesherren, insbesondere die Erzbischöfe von Mainz, wollten es sich auch als J agdgebiet reserviert halten. Auf diese Weise war eine Grenze zwischen 
zwei Territorien eine ziemlich starke Grenze, es gab über diese Grenze hinweg kaum einen Handel oder andere Wirtschaftsbeziehungen, aber auch keine Heirat und auch sonst kaum K ontakte. Deshalb ist es nicht erstaunlich, dass sich innerhalb solcher Territorien Traditionen des Sprechens heraus bilden, die nahezu alle sprachlichen Ebenen betreffen und die im Bereich des Lexikons über den kulturellen Wortschatz im engeren Sinne hinausgehen. Der Terminus ,Kultur' bezeichnet im Sinn der Ethnologie das Repertoire von "Traditionen [...], die eine Generation an die nächste" weiter gibt, „und zwar nicht auf dem Weg über die elterlichen Gene, sondern dadurch, dass die J üngeren von ihren Eltern und Gefährten " lernen, „wie man bestimmte Dinge macht" ( $H$ ar $r$ is 1996, S. 65). Es ist schon Schulbuchwissen geworden, zwischen materieller, sozialer und ideeller Kultur zu unterscheiden, „ohne daß sich allerdings scharfe Grenzen ziehen ließen" (Häuß I er N ow ot ny 1957, S. 149). Und ich möchte auch die schon genannten Traditionen des Sprechens ebenfalls zu den kulturellen Phänomenen rechnen, sodass die politischen Räume auch zu Kulturräumen werden. Ein Kulturraum entsteht also durch die „Verbreitung von durch Menschen getragenen Dialekt- bzw. Kulturerscheinungen im Raume" (G r ober -GI ück 1982, S. 92).

Nehmen wir als ein Beispiel die Bezeichnungen für die Zeitbestimmung , in diesem J ahr' (siehe K arte 4 im Anhang).

Die Karte zeigt vor allem zwei lexikalische Typen: Im Osten findet sich das Adverb heuer, in Westen das substantivische Syntagma das I ahr. Dabei überwiegt im gesamten Untersuchungsgebiet der Typ das J ahr. „Die Großraumkarten des DWA und des WDU zeigen, dass ,heuer' weite Teile des gesamten oberdeutschen Sprachraums einnimmt und sich großräumig im Ostmitteldeutschen fortsetzt." (B ayha 2002, Karte 2). Unsere Karte zeigt allerdings, dass im Unterostfränkischen das Adverb durch das substantivische Syntagma eine starke K onkurrenz bekommen hat, sodass an manchen Orten die Gewährspersonen beide Ausdrücke als Antwort gegeben haben.

Mit anderen Worten: Die sprachlichen Traditionen, die die beiden Teilareale über lange Zeit hin gekennzeichnet haben, beginnen sich aufzulösen. Es setzt sich die im Westen gültige Form das J ahr durch. Es entsteht ein neues, ein größeres Areal, das sich mit der neuen, der relativ jungen Verwaltungseinheit, dem Regierungsbezirk Unterfranken, deckt. Auffällig ist dabei, dass sich nicht die Standardform dieses J ahr durchsetzt, sondern eine Form, die stärker dialektal geprägt ist. Dies ist umso auffälliger, als J ürgen Eich of f (1977, K arte 42) für die U mgangssprachen die standardnahe Form dieses $\mathrm{J}$ ahr bucht (siehe Karte 5 im Anhang). 
Es geht also nicht um einen Mundartabbau, sondern um einen Mundartumbau: Wie ich schon an anderer Stelle auf der Basis phonologischen Materials beschrieben habe (vgl. W ol f 2003), bilden sich neue dialektale Formen oder setzen sich dialektale Formen größerräumig durch. Aus kleinräumigen, oft auch aus lokalen Dialekten werden größerräumige $V a$ rietäten, die aber nicht standardnäher sein müssen.

$\mathrm{Ob}$ die weiteren von uns erhobenen Zeitbestimmungen diesem Beispiel folgen, kann - noch - nicht gesagt werden. Die K arte , heute früh' (B ayh a 2002, K arte 9) etwa zeigt von vorneherein ein etwas anderes Bild (siehe Karte 6 im Anhang).

Hier ist ebenfalls ein Ost-West-Gegensatz zu bemerken: Das Rheinfränkische ist durch den Typ heute morgen gekennzeichnet, während das Unterostfränkische in weiten Bereichen heute früh aufweist. Allerdings finden wir im Fuldaer Übergangsstreifen und im Henneberger Raum ebenfalls den Typ heute morgen. Das will sagen, dass mitteldeutsche Formen eben nicht nur im Gebiet des ehemaligen Erzstiftes Mainz, sondern auch in anderen Gebieten im heutigen Unterfranken anzutreffen sind. Die Karte zeigt, dass im Westen des Untersuchungsgebietes ebenfalls schon die Form heute früh begegnet. Dies kann als weiteres Anzeichen für das Entstehen eines größeren Areals gedeutet werden, wobei sich anscheinend die W ürzburger Prestigeform durchzusetzen beginnt.

Wieder anders ist die Raumbildung bei ,gestern' (siehe Karte 7 im Anhang). Auch hier verwendet das ehemals mainzische Gebiet weitgehend einheitlich den Typ gestern, der aber auch den Süden des U nterostfränkischen, insbesondere das Gebiet östlich der Schweinfurter Staffel kennzeichnet. Nördlich von Würzburg herrscht der Typ nächten vor, ebenfalls mit einigen Streubelegen von gestern. Hier zeigt sich eine durchgehende südliche Form, während nächten sich nach Norden ins Thüringische fortsetzt.

Fassen wir unsere Detailbeobachtungen zu drei Zeitbezeichnungen zusammen: Das Untersuchungsgebiet konstituiert sich zunächst aus zwei ziemlich stabilen Kernräumen, dem Aschaffenburger und dem Würzburger Raum. Dazwischen liegt der Mainzer Übergangsstreifen, und auch die übrigen unterostfränkischen Teile sind als Übergangsgebiete zu interpretieren, die sich je nach sprachlichen Phänomenen zu unterschiedlichen Räumen hin ausrichten. Das Bild, das das Unterostfränkische bietet, dürfte zum Einen seine U rsache darin haben, dass der unterostfränkische Dialektraum sich bei Weitem nicht zur Gänze mit dem Territorium des Hochstifts Würzburg deckte. 
Eine Karte aus dem ,Bayerischen Geschichtsatlas' (Spindler 1969, S. 25) zeigt eindrucksvoll, dass das Gebiet des Hochstifts Würzburg von vielen kleineren Territorien, z. T. von kleinen Enklaven durchsetzt ist. Demgegenüber ist der sogenannte mainzische Teil Frankens ein weitgehend zusammen hängendes Areal. Gleichzeitig sehen wir, dass das Gebiet, das wir ,Fuldaer Ü bergangsstreifen' genannt haben, zum K loster Fulda gehörte. So gesehen, wird - ich bleibe im kulturmorphologischen Erklärungsrahmen - "die vereinheitlichende K raft politischer Verwaltungsbezirke, kirchlich und konfessionell bestimmte[r] Bindungen" (Grober GI ück 1982, S. 92) deutlich sichtbar. In diesem Zusammenhang wird es plausibel, das sich auch in wortgeographischer Hinsicht sowohl der Würzburger Raum als auch der Aschaffenburger Raum als stabile Kernräume darstellen.

Die territorialen Verhältnisse im Untersuchungsgebiet dürften aber als einzige Erklärung für unseren Befund nicht ausreichen. Im Zusammenhang mit den Erhebungen zum ,Sprachatlas von Unterfranken' wurden auch mehrere außersprachliche Fragen gestellt. Die Antworten auf die Frage ,Wohin geht man in die Stadt? ergeben ein ziemlich eindeutiges Kartenbild (L ange 1999, K arte 17; siehe Karte 8 im Anhang).

W ir sehen hier drei Zentren: Aschaffenburg, Würzburg und Schweinfurt, wobei Schweinfurt in unserem Zusammenhang keine Rolle spielt. Die Stadt Schweinfurt ist nach allen Erhebungen nicht Sprachraum bildend, was seinen Grund darin haben könnte, dass die Funktion als ein städtisches Zentrum erst jüngeren Datums ist; in der frühen Neuzeit war Schweinfurt als freie R eichsstadt protestantisch und somit eine Enklave in katholischem Umland.

Kehren wir zu den beiden wortgeographischen Kernräumen zurück. Der Teilband des SUF, der sich mit den Bereichen ,Zeiteinteilung und Grußformeln, Essen und Trinken, Brot und Brotbacken, Hausarbeit, Spielen und Spielzeug, K leinwörter (z. B. Indefinita, Adverbien)' (B ay ha 2002) befasst, enthält in seiner Dissertationsform eine Zusammenfassung, die sich besonders mit den Ost-West-Differenzen befasst. Sie liefert dazu zwei Tabellen, 1. Lexikalische Unterschiede am Mainzer Ü bergangsstreifen und 2. Lexikalische Unterschiede am Mainzer und Fuldaer Übergangsstreifen:

1.

\begin{tabular}{|l|l|l|}
\hline \multicolumn{3}{|l|}{ Unterschiede am Mainzer Übergangsstreifen } \\
\hline Lemma & Westl. Ausdruck & Östl. Ausdruck \\
\hline dieses J ahr & das J ahr & heuer \\
\hline Abschiedsgruß & adieu & ade \\
\hline
\end{tabular}




\begin{tabular}{|l|l|l|}
\hline \multicolumn{3}{|l|}{ Unterschiede am Mainzer Übergangsstreifen } \\
\hline A bendessen & Nachtessen & Abendessen \\
\hline Brösel & B rosam & B rösel \\
\hline Ofenschieber & Schießer & Schaufel, Schüssel \\
\hline Wollknäuel & K lüngel/Klingel & Kläue \\
\hline $\begin{array}{l}\text { nähen } \\
\text { (neues K leidungsstück) }\end{array}$ & nähen & flicken \\
\hline Wäsche klar spülen & auswaschen, flödern & lühen \\
\hline Wäscheleine & Leine & Seil \\
\hline Wäschekorb & Mande & K orb \\
\hline Murmel & Klicker & K ugel \\
\hline
\end{tabular}

2.

\begin{tabular}{|l|l|l|}
\hline \multicolumn{3}{|l|}{ Unterschiede am Mainzer und Fuldaer Übergangsstreifen } \\
\hline Lemma & Westl. Ausdruck & Östl. Ausdruck \\
\hline früher Winter & früher Winter & balder Winter \\
\hline in den April schicken & hinein den A pril schicken & in den April schicken \\
\hline heute A bend & heute Abend & heute abends \\
\hline heute Morgen & heute M orgen & heute früh \\
\hline $\begin{array}{l}\text { Zwischenmahlzeit am } \\
\text { Vormittag }\end{array}$ & Vesper & B rotzeit \\
\hline Bonbon & Guts (+Diminitivsuffix) & Leckerlein, Zuckerlein \\
\hline lutschen & lutschen, suckeln & züllen, schnullen \\
\hline Bäcker & Bäcker & Beck \\
\hline Vorteig bereiten & einmären & einmachen, einlegen \\
\hline Teigrest vom letzten Backen & Sauerteig & Deisem, H efel \\
\hline $\begin{array}{l}\text { Instrument, mit dem man } \\
\text { die Glut im Ofen verteilt }\end{array}$ & K ratze-r & Scharre-r \\
\hline Wäscheklammer & K lammer, Zwickel, Pflock & Zwicker \\
\hline Puppe & Puppe & Docke \\
\hline solange & solange & derweil \\
\hline
\end{tabular}

Wir sehen, dass diese Differenzen aus ganz unterschiedlichen Lebens- und Sachbereichen stammen. Von $52 \mathrm{~K}$ arten weisen 26, also genau die Hälfte, einen Ost-W est-Gegensatz auf. Allerdings ist dies nicht nur ein Gegensatz Mainz - Würzburg. Die zweite Tabelle, die auch das Material am Fuldaer Übergangsstreifen enthält, basiert auf fünfzehn Karten. Das heißt, dass etwas mehr als die Hälfte (58 \%) über Mainzisches Territorium hinaus weisen.

Wir können es uns einfach machen und einfach auf den Gegensatz Mitteldeutsch - Oberdeutsch verweisen. Dies scheint mir aber keine Lösung des Problems zu sein. Wir sind es gewohnt, die Sprachräume aufgrund von phonologischen $\mathrm{K}$ riterien zu definieren, und wir ordnen diesen Räumen dann auch lexikalische Merkmale zu. Nun können wir die funda- 
mentalen phonologischen Kennzeichen der Sprachräume teilweise weit in die Sprachgeschichte zurückführen, die hier vorgestellten lexikalischen Differenzen können aber nicht als ,elbgermanische ${ }^{\star}$ Eigenarten dargestellt werden; sie sind weitaus jünger und am ehesten als Ergebnisse der eingangs erwähnten Traditionen des Sprechens in bestimmten Arealen zu erklären. Angesichts des B efundes aus 26 W ortatlaskarten müssen wir aber annehmen, dass solche Areale durchaus größer sein können und sind als spätmittelalterliche oder frühneuzeitliche Territorien. Nicht eingehen kann ich zudem auf die Tatsache, dass die einschlägigen Isoglossen zum Teil ziemlich weit auseinander gehen ( $K$ arte aus B ay ha 2002, S. 128, siehe Karte 9 im Anhang):

Dies ist keine neue Erfahrung. Gleichzeitig sehen wir noch etwas W eiteres: Im Mainzer Übergangsstreifen bündeln sich die Isoglossen, was dieses Gebiet als prototypisches Übergangsgebiet ausweist. Im Norden teilt sich dann dieses Bündel, ein Teil geht nach Nordosten in den Fuldaer Übergangsstreifen, der andere setzt sich, was auf unserer Karte nicht sichtbar ist, direkt nach Norden fort. Das Übergangsgebiet wird zu zwei schwächeren Übergangsstreifen in dem Sinn, dass sie weniger Isoglossen enthalten. Andererseits ist die Areal bildendende K raft der beiden Kernräume so ,stark', dass sich zwischen innen ein ebenfalls starkes Übergangsgebiet etablieren kann. Es scheint nördlich des Aschaffenburger und des Würzburger Raumes nicht gleich wieder so ,starke' Räume zu geben.

Offen bleibt die Frage, ob sich alle bislang aufgeführten lexikalischen Differenzen auf kulturelle Unterschiede zurückführen lassen. U nser einleitendes M ost-Beispiel wäre wohl ein solches. Dabei ist aber auch zu beachten, dass der Wortgebrauch z. T. hier dem folgt, was in der Rheinregion bei Mainz üblich ist (vgl. K I eiber 1996, K arte 109).

Allerdings bezeichnet am R hein das W ort M ost den ungegorenen Saft, dies im U nterschied zum unterfränkischen Gebiet am Untermain.

Aus dem Bereich der Landwirtschaft, in Sonderheit aus der Viehzucht, finden sich weitere derartige Beispiele. Als Beispiele wähle ich das ,Gebären von J ungen' (nach G r imm 2002, S. 124), für das folgende Verben bzw. verbalen Syntagmen erhoben worden sind:

\begin{tabular}{|l|l|l|l|}
\hline Gattung & $\begin{array}{l}\text { Bezeichnung für das } \\
\text { Jungtier }\end{array}$ & $\begin{array}{l}\text { Gattungsspezifische } \\
\text { Bezeichnung für das } \\
\text { Gebären }\end{array}$ & $\begin{array}{l}\text { Gattungsunspezifische } \\
\text { Bezeichnung für das } \\
\text { Gebären }\end{array}$ \\
\hline Rind & K alb & kalben, kälbern & $\begin{array}{l}\text { hecken, } \\
\text { jung machen }\end{array}$ \\
\hline
\end{tabular}




\begin{tabular}{|l|l|l|l|}
\hline Ziege & Geiß, Zicke, Häpper & zickeln & $\begin{array}{l}\text { hecken, } \\
\text { Junge machen, } \\
\text { jung machen, } \\
\text { jungen/jüngen }\end{array}$ \\
\hline Schaf & $\begin{array}{l}\text { Lamm (Dim.), } \\
\text { Schaf (Dim.), } \\
\text { Bätzer (Dim.) } \\
\text { Hammelein }\end{array}$ & lammen & $\begin{array}{l}\text { hecken, } \\
\text { J unge machen, } \\
\text { jung machen } \\
\text { jungen/jüngen } \\
\text { werfen }\end{array}$ \\
& $\begin{array}{l}\text { Sau (Dim.) } \\
\text { Ferkel }\end{array}$ & ferkeln, \\
& feren & $\begin{array}{l}\text { hecken, } \\
\text { J unge machen, } \\
\text { jung machen, }\end{array}$ \\
& & & $\begin{array}{l}\text { Junge kriegen, } \\
\text { jungen/jüngen, } \\
\text { werfen, } \\
\text { schütten }\end{array}$ \\
\hline
\end{tabular}

Der wortgeographische Befund sieht folgendermaßen aus (wobei ich diesmal keine K arte präsentiere, sondern ein Modell, das nur die relative Lage der einzelnen $R$ äume wiedergibt):

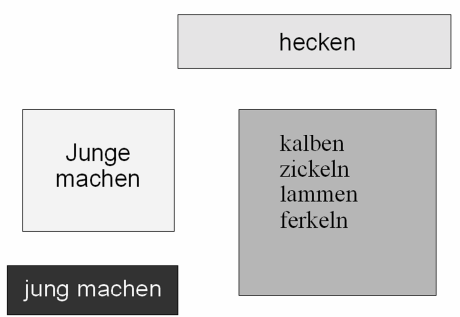

Abb 1: Modell ,gebären'

- Im nördlichen Henneberger Raum, der als Übergangsraum zum Thüringischen und Osthessischen anzusehen ist, wird fast durchgehend das Verbum hecken verwendet.

- Im Aschaffenburger Raum gilt nahezu durchweg das verbale Syntagma $J$ unge machen.

- Im südlichen Amorbacher Raum sagt man jung machen.

- „Ausnahmslos gattungsspezifische B ezeichnungen finden sich im zum oberdeutschen Sprachraum gehörigen Unterostfränkischen belegt." (G r imm 2002, S. 124). 
Es lässt sich vermuten, dass im Unterostfränkischen die Viehzucht eine viel wichtigere Rolle spielt als in den anderen Arealen, sodass die Terminologie viel differenzierter ist. Auffällig ist, dass sich ein so eindeutiges Bild bei anderen einschlägigen Bezeichnungen, etwa für die Brunst, nicht bietet.

Unbeantwortet bleiben muss die Frage, ob die Konfession die Wortgeographie beeinflusst. Man führt im phonologischen Bereich gerne an, dass in protestantischen Orten Entrundungen (Frösch $>$ Fresch) zu beobachten sind. Unser Material bietet im lexikalischen Bereich keine derartigen Hinweise. Lediglich auf ein einschlägiges $P$ hänomen sind wir gestoßen: In protestantischen Orten in der Rhön wurde in Informantenkommentaren der Gruß Grüß Gott als typisch katholisch bezeichnet, und in einigen wenigen ebenfalls protestantischen Orten im Süden des Untersuchungsgebietes, im Bereich der protestantischen Grafschaft Castell, wurde als üblicher Gruß Guten Tag in einem eindeutigen Grüß-Gott-U mfeld als Antwort gegeben.

\section{Zusammenf assung}

Das genaue Lesen einiger Wortkarten des SUF hat im heutigen Regierungsbezirk Unterfranken zwei wortgeographische Kernräume ergeben, die sich mit den Zentren zweier Territorien bzw. Teilterritorien identifizieren lassen. In diesen Territorien haben sich, wie gesagt, Traditionen des Sprechens heraus gebildet, die sicherlich einen wichtigen Teil der sozialen Kultur, mithin der Kulturgeschichte dieser Räume ausmachen. Dass diese Traditionen des Sprechens sich auf die materielle Kultur, insbesondere auf wesentliche Teile der Wirtschaftskultur beziehen, stützt unsere Annahme von solchen kulturräumlichen Gegebenheiten. Gleichzeitig ergibt sich, dass wortgeographische Areale gut kulturmorphologisch interpretiert werden können. Der Ansatz ,Wörter und Sachen' kann die kulturmorphologische Methode unterstützen.

\section{Zitierte Liter at ur}

B ad. W b. (1997): Badisches Wörterbuch Bd. 3. bearb. von Gerhard W. B aur . Lahr.

B ayha, Karin (2002): Wortkarten des Sprachatlas von Unterfranken zu den Themenbereichen ,Zeiteinteilung und Grußformeln, Essen und Trinken, Brot und Brotbacken, Hausarbeit, Spielen und Spielzeug, K leinwörter (z. B. Indefinita, Adverbien)'. Diss. W ürzburg. 
D W A = Deutscher W ortatlas. Von Walther M it zka [Bd. 5ff. von Walther M it zka und Ludwig Erich Schmitt ] Bde. 1-20. Gießen 1951ff. Bde. 21 u. 22 hrsg. v. Reiner H il debrandt. Gießen 1978, 1980.

Fischer, Roman (1995): Das Untermaingebiet und der Spessart. In: Peter K ol b, ErnstGünter K r enig (1995), S. 393-452.

Frankf. W b. (1988); Frankfurter Wörterbuch Bd. 4. Bearb. von Hans-Otto Schembs, Günther V ogt. Frankfurt (Main).

G r imm, Manuela (2002): Wortkarten des Sprachatlas von Unterfranken zu den Themenbereichen, Rindvieh, Rübenbau, Milch und Milchverarbeitung, Ziege und Schaf, Schwein und Hausschlachten, Gelügel und weitere Haustiele. Diss. Würzburg.

Gr ober -GI ück, Gerda (1982): Die Leistungen der kulturmorphologischen Betrachtungsweise im Rahmen dialektgeographischer Interpretationsverfahren. In: Dialektologie. Ein Handbuch zur deutschen und allgemeinen Dialektforschung. Hrsg. von Werner B esch, Ulrich K noop, Wolfgang Putschke, Herbert Ernst W iegand. Halbbd. 1. Berlin, New York, S. $92-113$.

$\mathrm{H}$ ar r is, Marvin (1996): Menschen. Wie wir wurden, was wir sind. München (=dtv 30530).

H äuß I er , Franz/N ow otny, Ernst (1957): Einführung in die Logik und Wissenschaftslehre und in die Grundprobleme der Philosophie. Wien.

Kl eiber, Wolfgang (1996): Wortatlas der kontinentalgermanischen Winzerterminologie. Tübingen.

K I uge, Friedrich (2002): Etymologisches Wörterbuch der deutschen Sprache. 24. Aufl. von Elmar Seebold. Berlin, New Y ork.

K ol b, Peter/K r enig, Ernst-Günter (Hrsg., 1995): Unterfränkische Geschichte Bd. 3. Würzburg.

K ol b, Peter/K r enig, Ernst-Günter (Hrsg., 1998): Unterfränkische Geschichte Bd. 4/1. Würzburg.

K ol b, Peter/K r enig, Ernst-Günter (Hrsg., 1999): Unterfränkische Geschichte Bd. 4/2. Würzburg.

Lange, Michael (1999): Probleme der „Einführungskarten“ für den Sprachatlas von Unterfranken. MA-Arbeit, Würzburg.

Schenk, Winfried (1995): Die mainfränkische Landschaft unter dem Einfluß von Gewerbe, Handel, Verkehr und Landwirtschaft. In: Peter K ol b, Ernst-Günter K renig (1995), S. 519-588.

Spindl er , Max (Hrsg., 1969): Bayerischer Geschichtsatlas. München.

Spr andel, Rolf (1978): Verfassung und Gesellschaft im Mittelalter. 2. Aufl. Paderborn, München, Wien, Zürich.

$W D U=$ Wortatlas der deutschen Umgangssprachen. Von J ürgen Eichhoff. Bd. 1. Bern, München.

W olf, Norbert Richard (2002): Regionen entstehen, indem die Leute miteinander reden. Oder: Regionalisierungsprozesse in rezenten Dialekten. In: Regionalität als Kategorie der Sprach- und Literaturwissenschaft. Hrsg. vom Instytut Filologii Germanskiej der Uniwersytet Opolski. Frankfurt (Main) u. a. (Oppelner Beiträge zur Germanistik 6), S. $97-110$. 



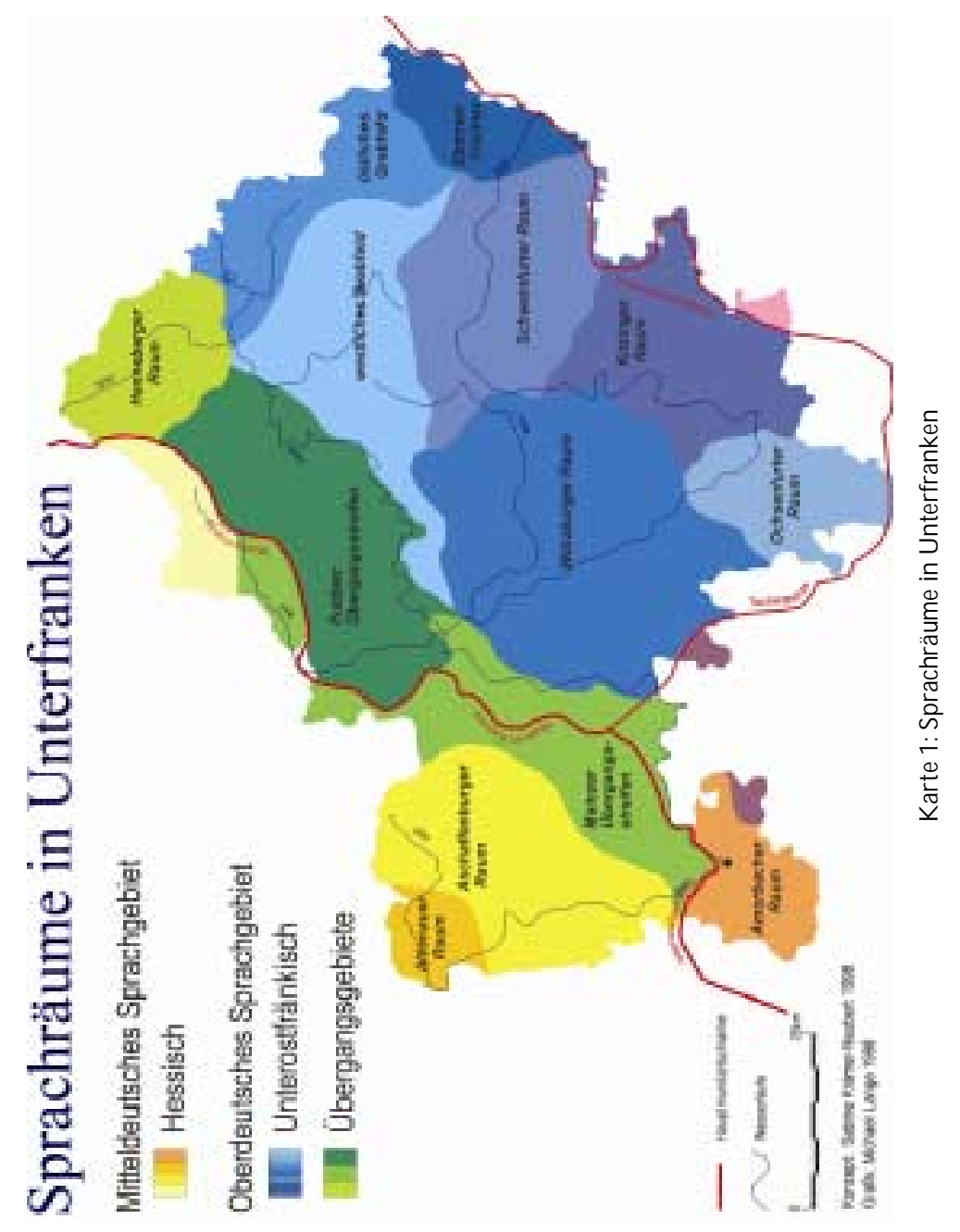




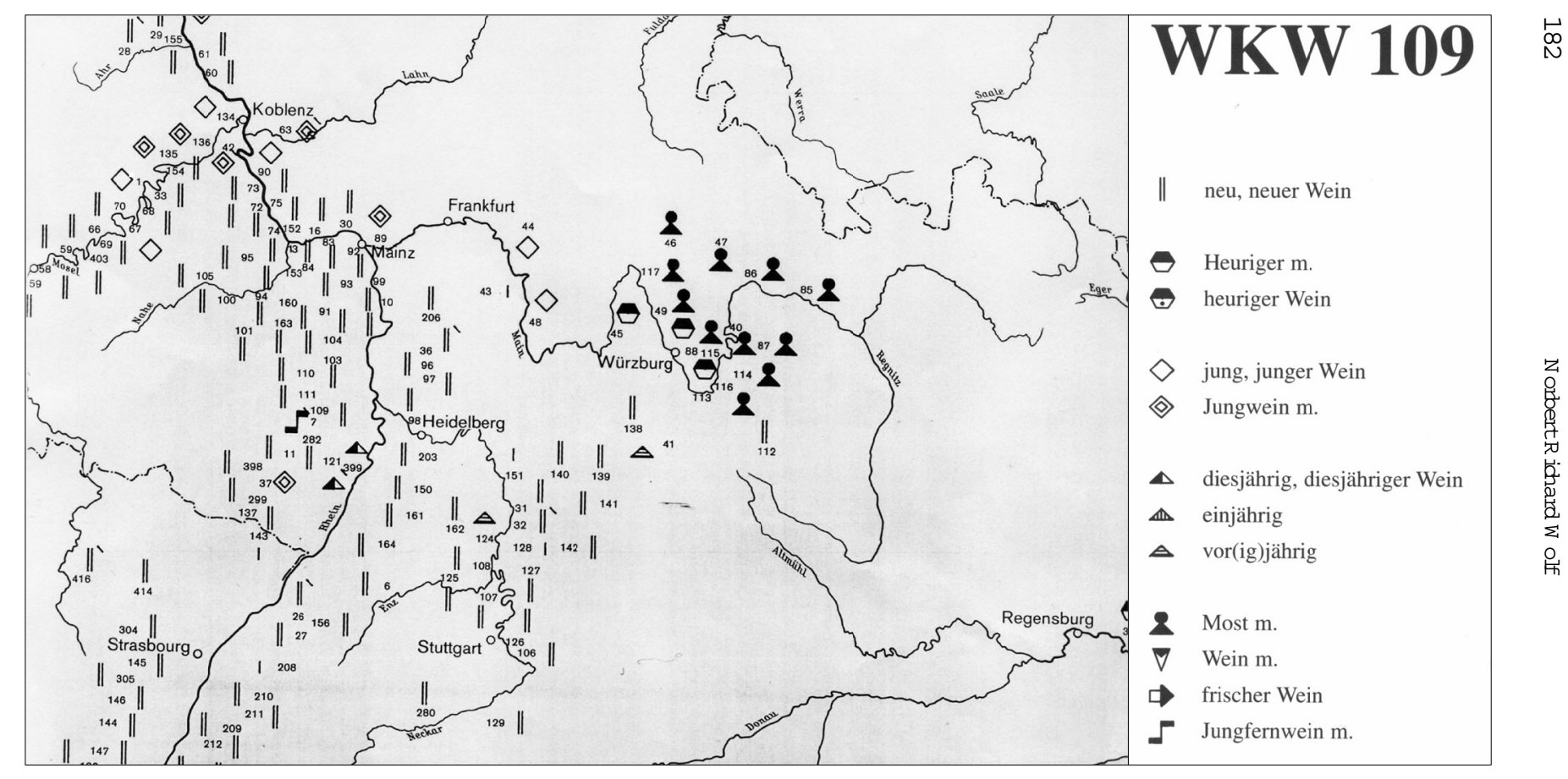

Karte 2 : D erW ein neuerEmte (aus K leiber 1996, Karte 109) 


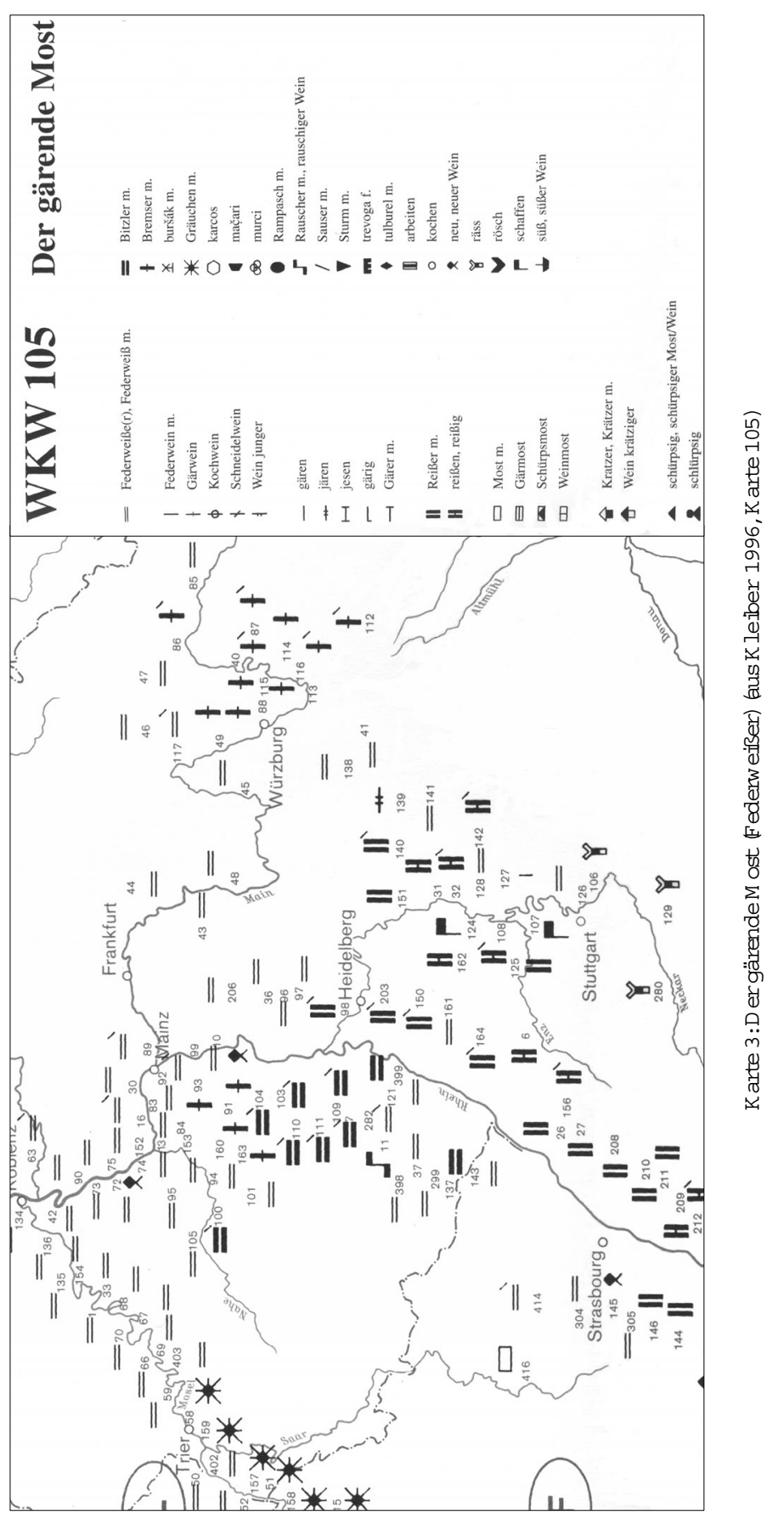




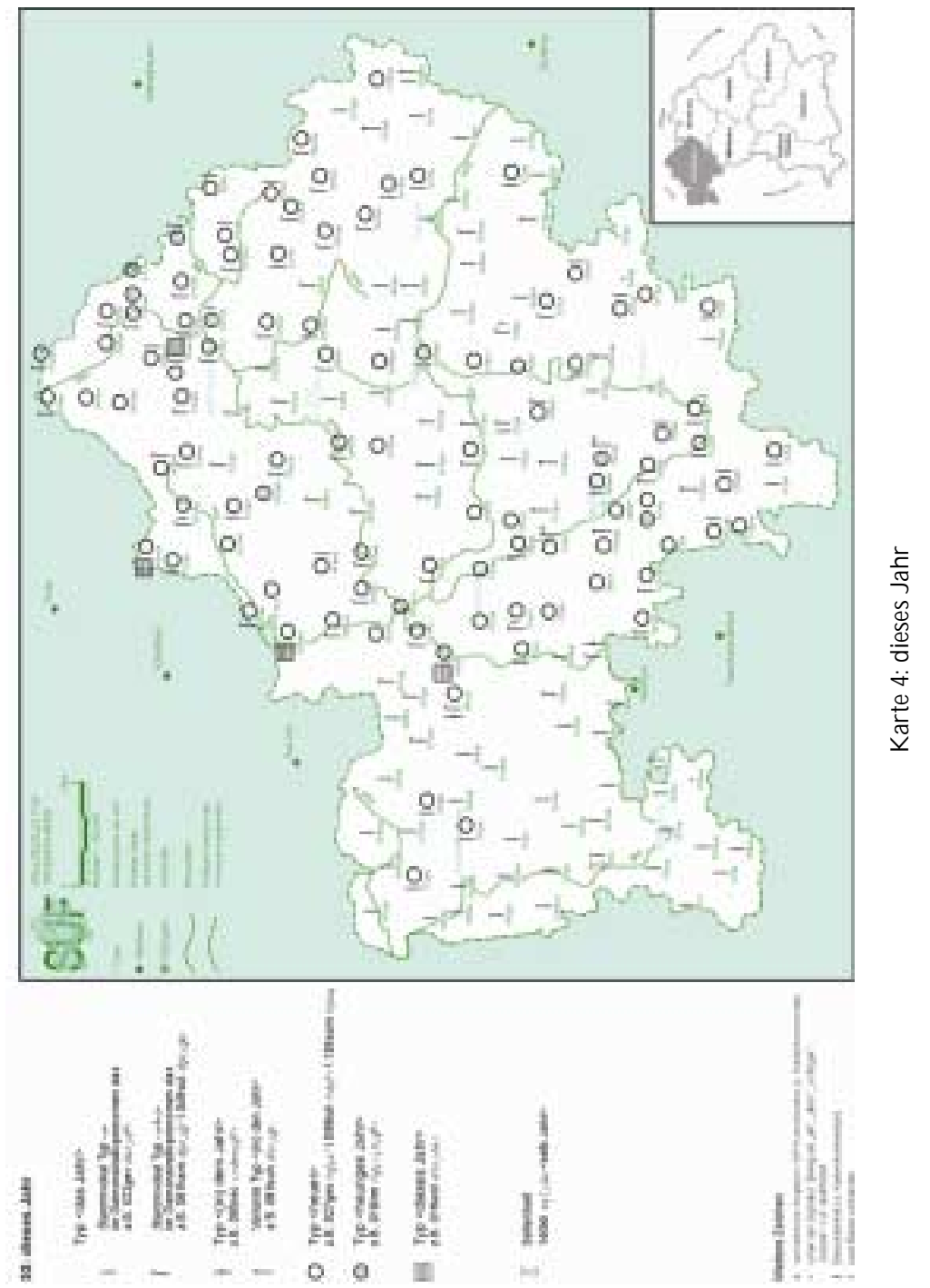




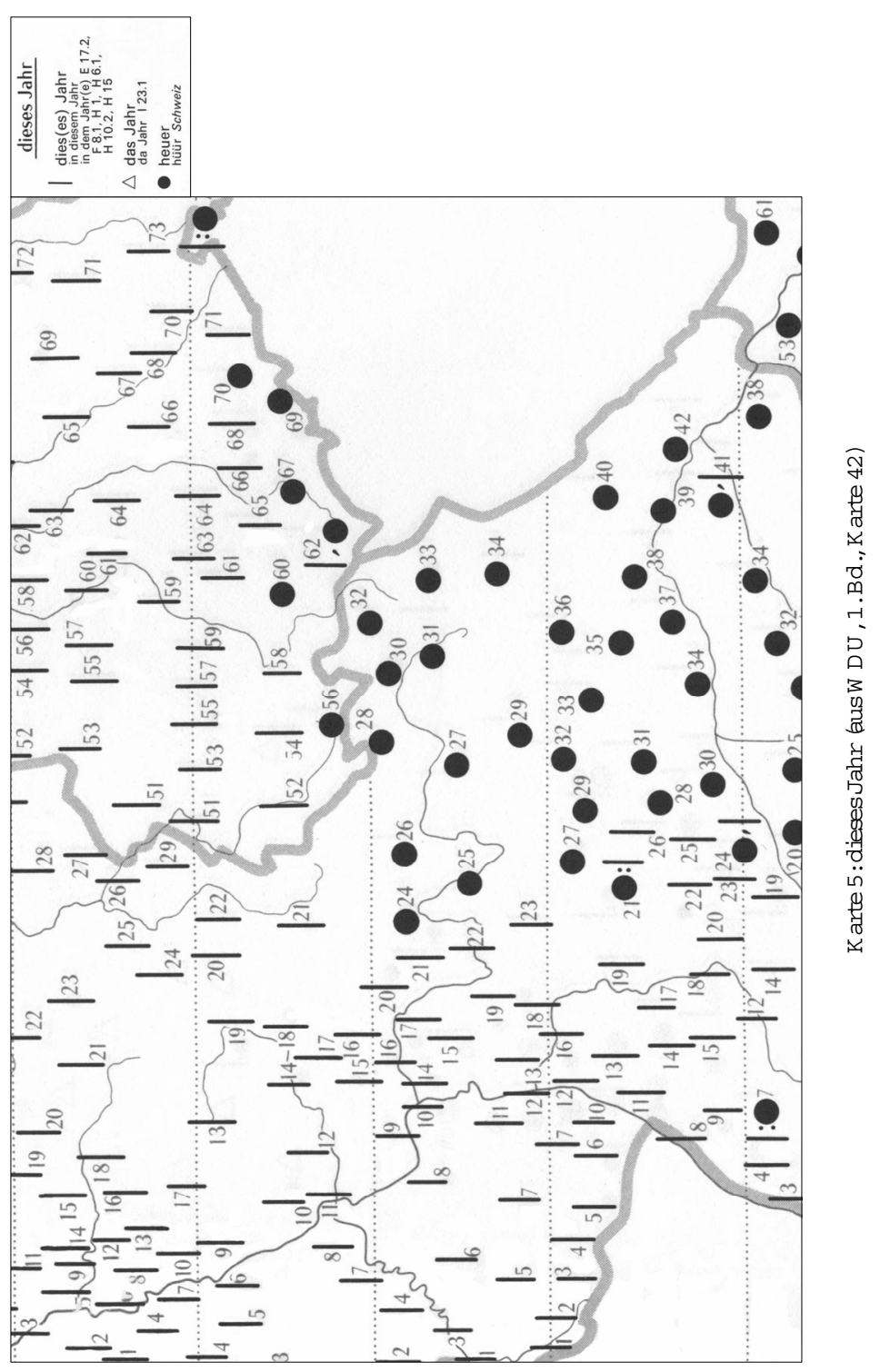




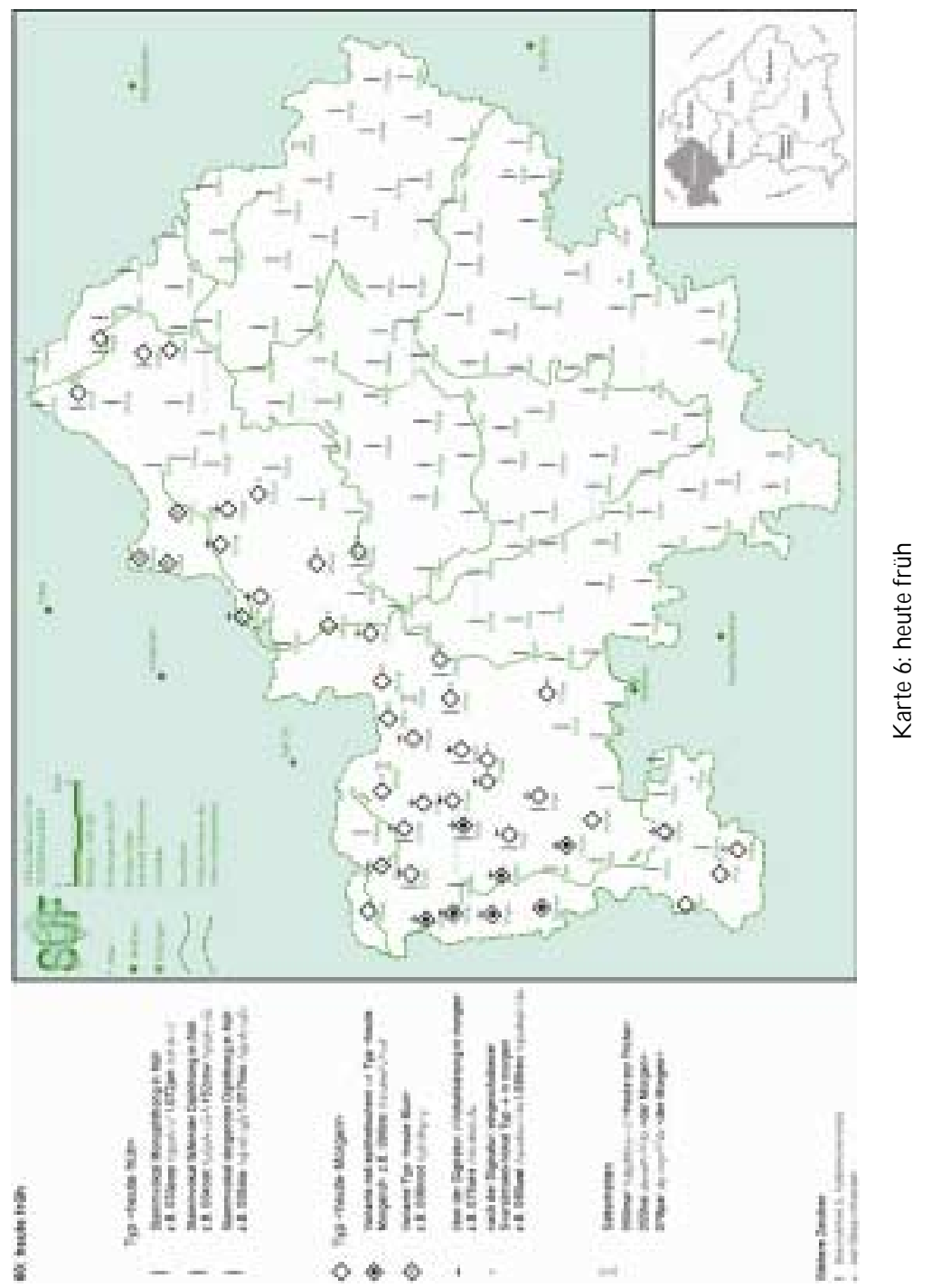




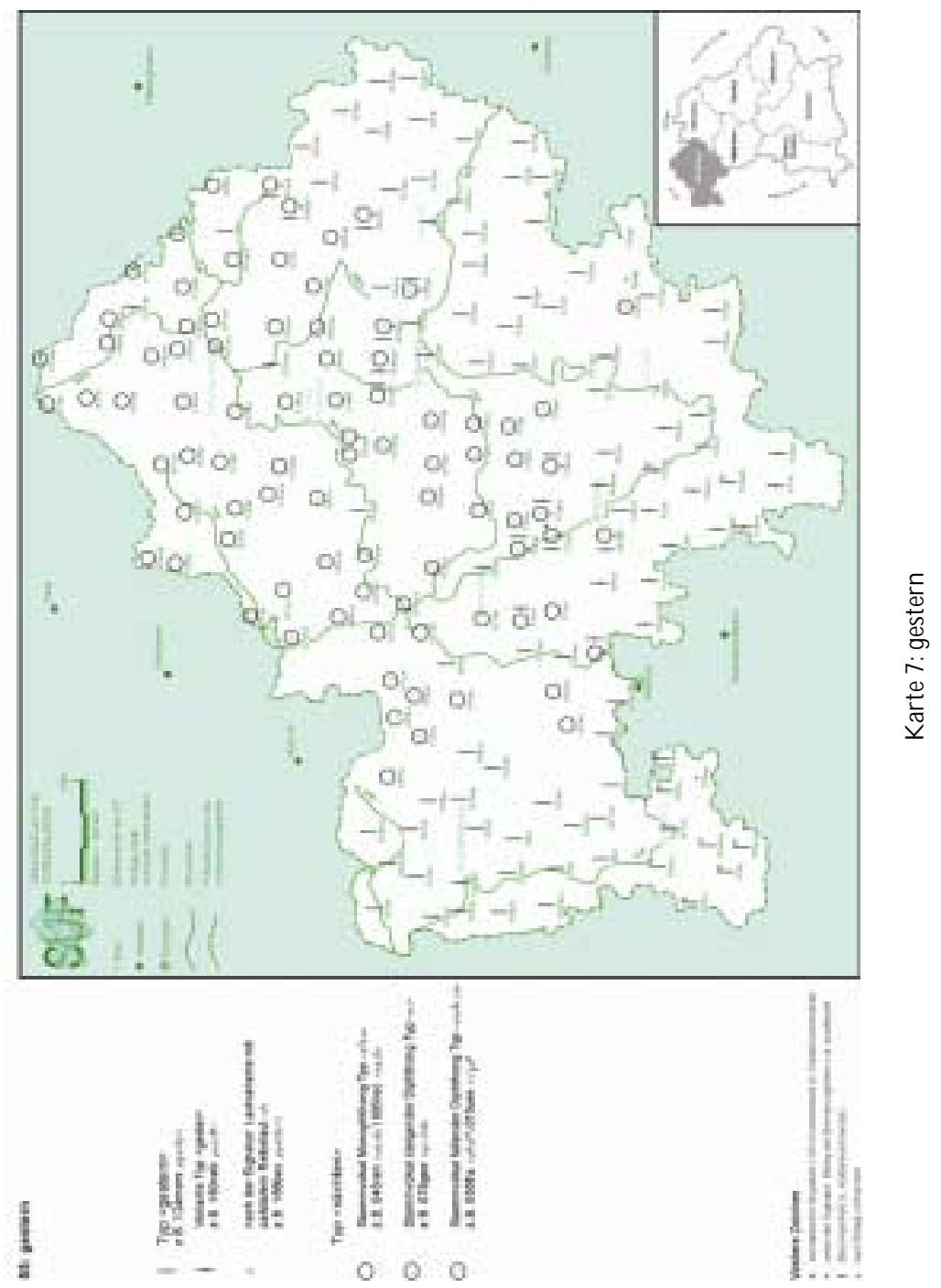




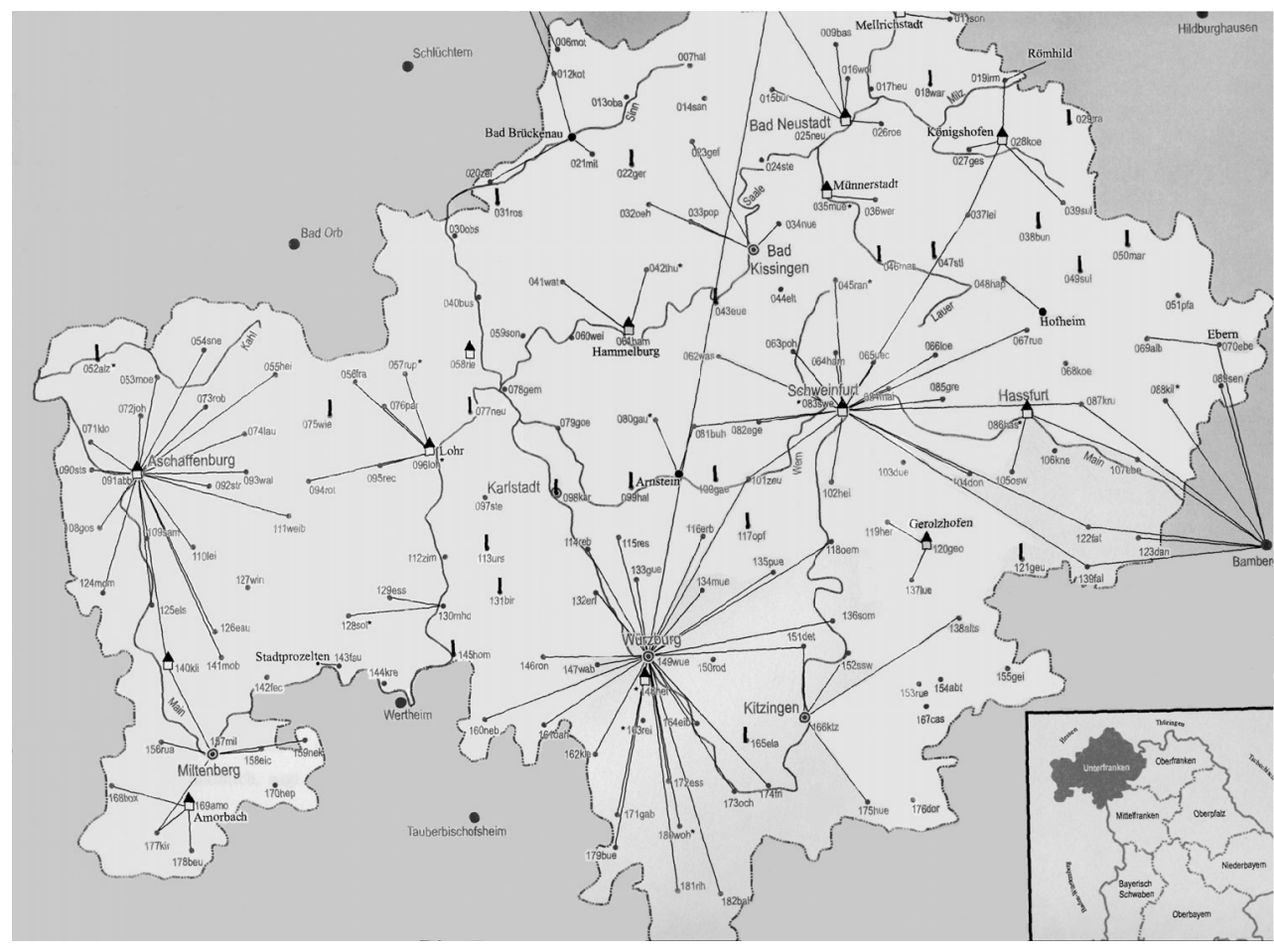

Karte 8:W ohin gehtm an in die Stadt? aus Lange 1999, Karte 17) 


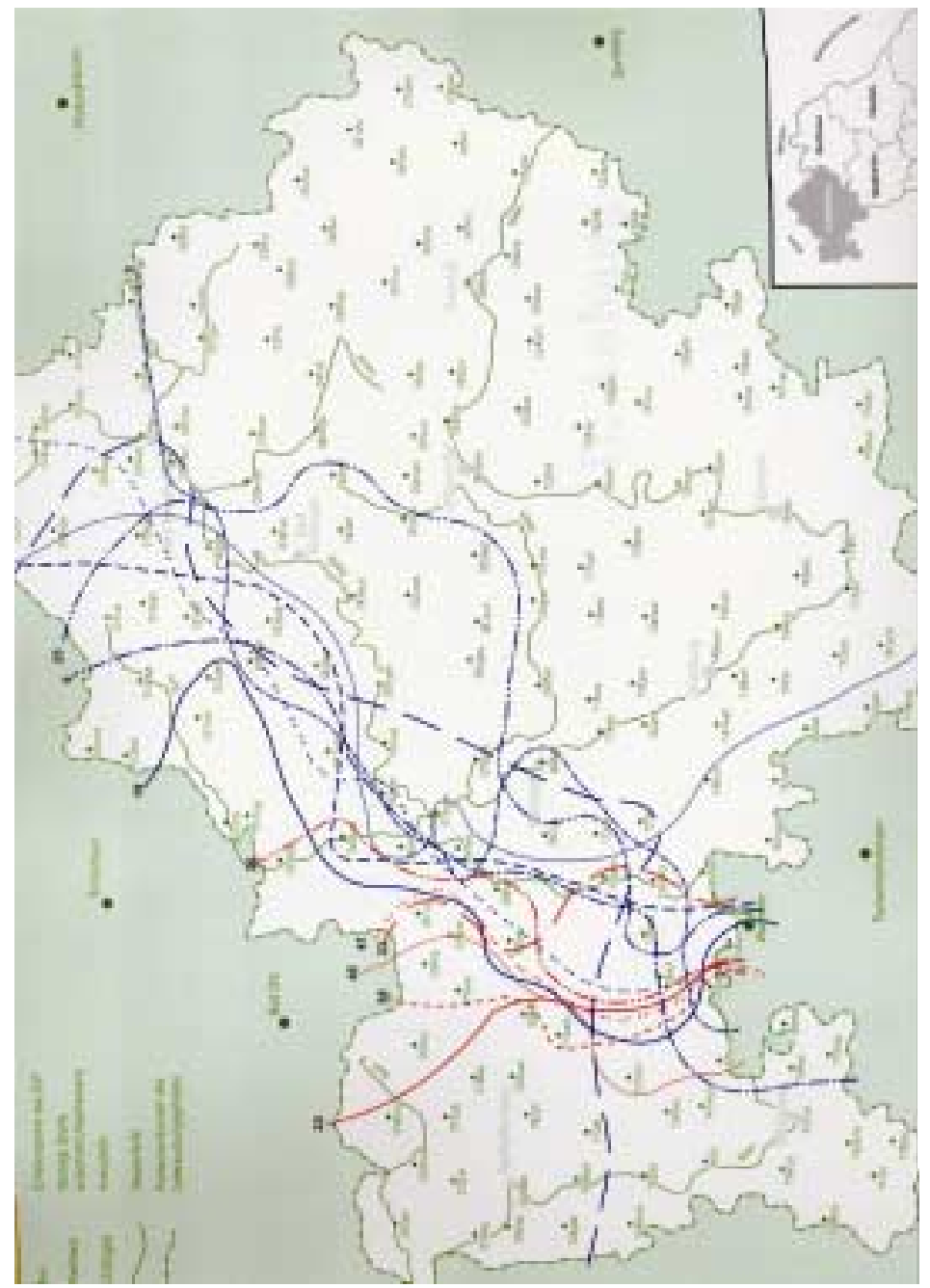

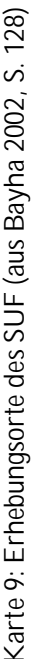


